\title{
Incidence of Propionibacterium acnes infection in orthopedic and trauma surgery
}

\author{
I Uckay ${ }^{1 *}$, A Agostinho ${ }^{1}$, C Landelle ${ }^{1}$, E Coppens ${ }^{1}$, G Cunningham¹', D Pittet $^{2}$ \\ From 3rd International Conference on Prevention and Infection Control (ICPIC 2015) \\ Geneva, Switzerland. 16-19 June 2015
}

\section{Introduction}

Propionibacterium acnes has been associated with late, smoldering and healthcare-associated infections of the shoulder and spine.

\section{Objectives}

The epidemiology of $P$. acnes with respect of other orthopedic locations and patient populations remains largely unknown.

\section{Methods}

Retrospective, single-center, descriptive and case-control studies of adult patients hospitalized for orthopedic infections from 2004-2014. We used only intraoperative microbiological samples and first clinical infection episodes. Cefuroxime (or vancomycine) was used for perioperative prophylaxis. Microbiological samples were incubated for a median of 5 days.

\section{Results}

$P$. acnes was isolated intraoperatively in only $37 / 2740$ (1.35\%) surgical procedures. A total of $22 / 37$ infections were monomicrobial. Overall, 665 surgical procedures (24\%) involved hardware/osteosynthesis material. $P$. acnes was more frequently identified during procedures in the presence compared with the absence (24/ 665 vs. $13 / 2075 ; p<0.01$ ) of hardware/foreign material. $P$. acnes was frequently associated with other skin commensals $(12 / 291$ vs. $25 / 2134 ; p<0.01)$ and involved the lumbar and shoulder regions. The proportion of $P$. acnes among all pathogens in the spine and shoulder were $8 \%$ and $6 \%$, respectively. In contrast, $P$. acnes was almost never identified ( $3 / 1021$ vs. $334 / 1719 ; p<0.01)$ among immune-suppressed patients, in foot infections, septic bursitis, native bone and joint infections, soft tissue abscesses, prosthetic joints, and tibia nails. By multivariate analysis adjusting for case-mix, the lumbar region (odds ratio $7.4,95 \%$ CI 1.2-46.3), the shoulder (OR 9.9, 1.6-60.1) and the presence of hardware (OR 8.2, 2.4-28.4) were significantly associated with $P$. acnes infection; while sex, age, immune-suppression and the administration of antibiotic therapy prior to intraoperative sampling were not.

\section{Conclusion}

In our institution, $P$. acnes is very rarely associated with clinical orthopedic infections. It is almost never responsible for infection below the lumbar spine level. P. acnes infections are associated with less inflammatory response than other infections. $P$. acnes is particularly associated with plate and spondylodesis infections.

\section{Disclosure of interest}

None declared.

\section{Authors' details}

1 Geneva University Hospitals, Geneva University Hospitals, Geneva,

Switzerland. ${ }^{2}$ Infection Control Program, Geneva University Hospitals, Geneva, Switzerland.

Published: 16 June 2015

doi:10.1186/2047-2994-4-S1-O28

Cite this article as: Uckay et al.: Incidence of Propionibacterium acnes infection in orthopedic and trauma surgery. Antimicrobial Resistance and Infection Control 2015 4(Suppl 1):O28. 